\title{
To code or not to code: rate optimality in node-capacitated networks
}

\author{
Ziyu Shao, Sidharth Jaggi, Shuo-Yen Robert Li \\ Department of Information Engineering \\ The Chinese University of Hong Kong \\ Shatin, N.T., Hong Kong \\ Email: \{zyshao6, jaggi, bobli\}@ie.cuhk.edu.hk
}

\begin{abstract}
Node-capacitated networks are networks in which the capacity constraint is put on every node. They have recently attract attention as a good model for Peer-to-Peer (P2P) overlay networks. Existing work gives results on networks with constraints of node upload capacities. In this paper, we consider networks with constraints on both node upload and node download capacity. For such networks, we investigate the rate optimality of routing versus network coding. In general, network coding achieves a larger rate region than routing. However, for some important communication scenarios, routing achieves the same rate region as network coding.
\end{abstract}

\section{INTRODUCTION}

Network coding [1] is the paradigm of allowing each node in a network to encode its input symbols. This is in contrast to the routing paradigm, where each node can only store and forward copies of its input symbols. Much prior work $[1,2,3]$ focused on models with capacity constraints put on edges rather than nodes. The latter model, however, is better fit for some communication scenarios such as P2P overlay networks. Therefore, the study of network coding with node-capacitated networks $[7,8,11,12]$ has generated much research interest. Existing work on node-capacitated networks mainly examines scenarios with only upload capacities constraints. The download capacity of nodes are assumed to be unbounded. However, in practice, each peer (node) has only limited download bandwidth, buffer and processing power. Thus we focus on node-capacitated networks with constraints on both node upload and node download capacities. We are concerned with the problem of rate optimality of routing versus network coding, i.e., whether routing achieves the same rate region as network coding or not.

In this paper, we have two types of results:

- Routing = Coding: routing achieves the same rate region as network coding for following communication scenarios.

1) Single-source unicast with general topology ${ }^{1}$

2) Single-source broadcast with general topology

3) Single-source multicast for some important classes of networks

4) Multi-source unicast with only intra-session network coding

\footnotetext{
${ }^{1}$ General topology here means no constraints are put on topology of network.
}

5) Multi-source broadcast with only intra-session network coding

6) Multi-source multicast for some important classes of networks

- Routing $\neq$ Coding: network coding achieves a larger rate region than routing for following communication scenarios.

1) Single-source multicast with general topology

2) Multi-source unicast with general topology

3) Multi-source broadcast with general topology

4) Multi-source multicast with general topology

Results in this paper has two forms:

- Theorem 1, 2, 3, 5 and 6 : direct extensions of results from max-flow-min-cut theorem [4], Edmonds' theorem [5] and butterfly networks [1,2].

- Theorem 4 and 7: main contributions.

\section{RELATED WORK}

For directed edge-capacitated networks, routing is rate optimal for single-source unicast and single-source broadcast with general topology. The former is implied by max-flow-min-cut theorem [4], and the latter is implied by Edmonds' theorem of packing spanning trees [5]. For single-source multicast, network coding achieves $[1,2,3]$ larger rate than routing.

For node-capacitated networks, existing results focus on networks with constraints on node upload capacity. For singlesource multicast (broadcast) with full-mesh topology, ${ }^{2}$ [6], [7], [8], [9] and [10] derived results of a similar flavor on rate-optimality of routing. For multi-source multicast with fullmesh topology, [11, 12] also derived results on rate-optimality of routing.

The remainder of this paper is structured as follows. In Section III, we introduce models and definitions used throughout this paper. In Section IV, our results are summarized, and sketch of proofs are provided. Detailed proofs of theorem 4 and related lemmas are provided in Section V. Conclusions are provided in Section VI.

${ }^{2}$ Full-mesh here means each node can communicate with all other nodes in the network, i.e., a complete graph. 


\section{Model And Definitions}

The node-capacitated network is represented by a directed graph $G=\left(V, E, C_{\text {out }}, C_{\text {in }}\right)$. Here $V$ denotes the set of nodes in the network, $E$ denotes the set of edges in the network, $C_{\text {out }}$ is a node upload capacity function for each node defined as $C_{\text {out }}: V \rightarrow \mathbb{R}^{+}$, and $C_{\text {in }}$ is a node download capacity function for each node defined as $C_{i n}: V \rightarrow \mathbb{R}^{+}$.

For convenience, the size of any set $\mathcal{K}$ is denoted by $|\mathcal{K}|$. The set of source nodes is denoted by $S$. For each source $s \in S$, let $R_{s} \subseteq V$ be the corresponding set of receivers for source $s$. Any node besides sources and receivers is called helper node. The set of helper nodes for source $s$ and the receiver set $R_{s}$ is denoted by $H_{s}$.

A unicast communication scenario is when each source $s$ has only one receiver, i.e., $\left|R_{s}\right|=1$. A broadcast communication scenario is when each source $s$ has no helpers, i.e., $\left|R_{s}\right|=$ $|V-\{s\}|=|V|-1$. A multicast communication scenario is when each source $s$ has more than one receivers and at least one helper, i.e., $1<\left|R_{s}\right|<|V|-1$.

For any node $v \in V$, let $\operatorname{In}(v)$ be the set of edges incoming to node $v$, and $O u t(v)$ be the set of edges outgoing from node $v$. Edge rate is defined as the sum of bandwidth occupied by all communication scenarios using the edge. Let $f(e)$ : $e \rightarrow[0, \infty)$ be the edge rate for edge $e$. We denote $f(E)=$ $[f(e), e \in E]$ as the length- $|E|$ edge rate vector defined on $E$.

Given a node-capacitated network, an edge rate vector is said to be feasible if for each node $v$ the sum of the edge rate of the edges leaving $v$ is at most $C_{\text {out }}(v)$, and the sum of the edge rate of the edges entering $v$ is at most $C_{i n}(v)$. That is, both node upload capacity constraints and node download capacity constraints are satisfied:

$$
\begin{gathered}
\sum_{e \in \operatorname{Out}(v)} f(e) \leq C_{\text {out }}(v) \\
\sum_{e \in \operatorname{In}(v)} f(e) \leq C_{\text {in }}(v)
\end{gathered}
$$

The rate of the source $s$ is denoted by $z_{s}$. Let $\vec{z}=\left\{z_{s}, s \in\right.$ $S\}$ denote the rate vector of the whole network. Thus the rate vector $\vec{z}$ is called achievable if there exists a feasible edge rate vector such that for each source $s \in S$, it is possible to transmit message $X_{s}$ from $s$ to its receiver set $R_{s}$, such that each receiver can accurately reconstruct $X_{s}$.

For a rate vector $\vec{z}$, define the set of all edge rate vectors achieving $\vec{z}$ as $F_{z}(E)=\{f(E): \vec{z}$ is achieved by $f(E)\}$.

Definition [11]: An edge rate vector $f_{z}^{*}(E) \in F_{z}(E)$ is irreducible with respect to $\vec{z}$ if there does not exist $f_{z}^{\prime}(e) \in$ $F_{z}(E)$ such that

$$
\sum_{v \in V} \sum_{e \in O u t(v)} f_{z}^{\prime}(e)<\sum_{v \in V} \sum_{e \in O u t(v)} f_{z}^{*}(e) .
$$

That is, the sum of all outgoing edge rates is minimal. Edge rate vectors which are not irreducible are called reducible. Irreducible edge rate vectors represent the most efficient edge rate assignment that achieves $\vec{z}$.

\section{Summary of Results}

Here we list our results for node-capacitated networks with both upload and download capacity constraints. Due to space limitation, most results (Theorem 1,2,3,5,6,7) are only provided with a sketch of proof, and full details can be found in [13]. The proof of Theorem 4 is given in detail in Section $V$.

Theorem 1. Routing is rate-optimal for single-source unicast with general topology.

This follows directly from max-flow-min-cut theorem for node-capacitated networks [4].

Theorem 2. Routing is rate-optimal for single-source broadcast with general topology.

This follows from Edmonds' theorem of packing spanning trees [5] for edge-capacitated networks.

Theorem 3. Routing is not rate-optimal for single-source multicast with general topology.

Node-capacitated butterfly network [13], which can be directly obtained from classical butterfly network $[1,2]$, provides the required example.

One of the main contributions of this paper is the following result.

Theorem 4. Given a single-source multicast scenario $\mathcal{M}=$ $\left(s, R_{s}, H_{s}, G, f u l l-m e s h\right)$. For the helper nodes satisfying the condition

$$
\begin{aligned}
& \min \left[\sum_{h \in H_{s}} C_{\text {in }}(h), \frac{1}{N} \sum_{h \in H_{s}} C_{\text {out }}(h)\right] \\
& =\sum_{h \in H_{s}} \min \left[C_{\text {in }}(h), \frac{1}{N} C_{\text {out }}(h)\right] .
\end{aligned}
$$

, routing is rate-optimal. The optimal multicast rate can be achieved by packing at most $|V|=1+\left|R_{s}\right|+\left|H_{s}\right|$ trees, each with depth at most 2.

Full details of the proof are provided in Section $V$.

Remark 1: Here we list some important scenarios satisfying condition (3):

- Empty set of helper nodes. It is a broadcast scenario. The work in [6], [8], [9], [10] falls into this category.

- All helper nodes either have only node upload capacity or have only node download capacity. The work in [7] falls into this category.

Theorem 5. Routing is not rate-optimal for multi-source unicast, multicast and broadcast with general topology.

Consider the network shown in Fig.1, which follows directly from the classic butterfly network $[1,2]$. Routing is shown to be not rate-optimal for multi-source unicast. Further, multisource unicast is a special case of multi-source multicast and multi-source broadcast. 


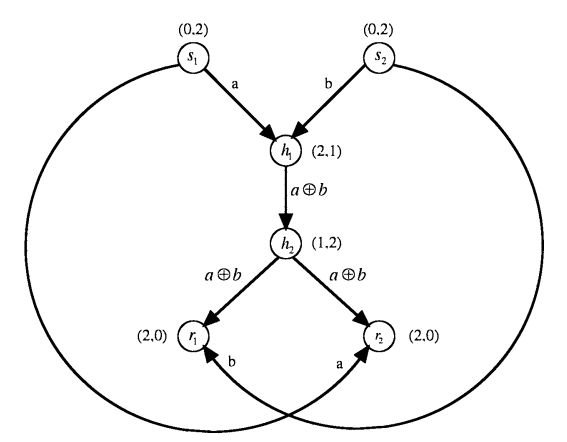

Fig. 1. Extended node-capacitated butterfly network. Each node is labeled with capacity pair: (download capacity, upload capacity). Each edge is labeled with network code achieving unicast rate 1 from $s_{1}$ to $r_{1}$ and $s_{2}$ to $r_{2}$ simultaneously. Routing can not achieve this because of bottleneck $h_{1} h_{2}$.

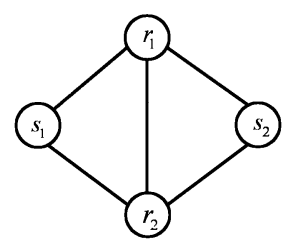

Fig. 2. P2P-Bridge Network. It consists of two sources ( $s_{1}$ and $\left.s_{2}\right)$, two receivers $\left(r_{1}\right.$ and $\left.r_{2}\right) . R_{s_{1}}=R_{s_{2}}=\left\{r_{1}, r_{2}\right\}$.

Theorem 6. For multi-source unicast and multi-source broadcast with general topology, routing achieves the same rate region as intra-session network coding.

When there is no coding across sessions belonging to different sources, the whole network can be regarded as the disjoint union of individual communication scenarios of each source. Then by theorem 1 and theorem 2, we get the desired result.

Another main contribution of this work is the following result.

Theorem 7. For multi-source multicast with general topology, the rate region obtained by cut-set bound is not the tight upper bound on achievable rate region.

The P2P-Bridge network, shown in Fig.2, provides the required example. The key reason is that upload capacity of $s_{2}$ (or $s_{1}$ ) will be useless for improving multicast rate of $s_{1}$ (or $s_{2}$ ). Further, in P2P-Bridge network, routing achieves the same multicast rate region as intra-session network coding.

Remark 2: For networks with full-mesh topology, rate regions obtained by cut-set are tight, just as theorem 4 and $[8,9,11]$ show. The key reason is that upload capacity of every node is useful because of full-mesh topology.

\section{Proof of Theorem 4}

Before the proof of theorem 4, we need the following two lemmas first.

We let $\mathcal{M}=\left(s, R_{s}, H_{s}, G\right.$, full-mesh $)$ denotes a singlesource multicast communication scenario for network $G$ with full-mesh topology, consisting of a single-source $s$, a receiver set $R_{s}$, and a helper set $H_{s}$.

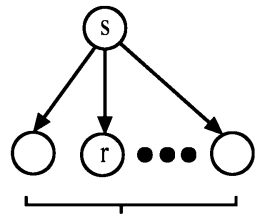

Type-1 Tree

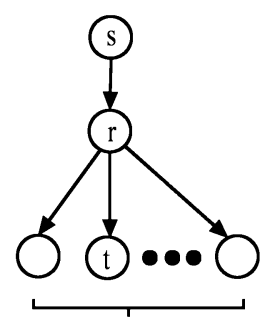

Type-2 Tree

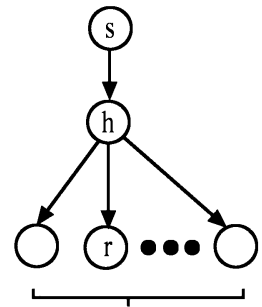

Type-3 Tree
Fig. 3. $[7,11]$. Three types of routing trees. $s \in S, h \in H_{s}$ and $r, t \in R_{s}$.

For convenience, we let $N=\left|R_{s}\right|$ and

$$
\begin{aligned}
& \delta=\frac{\sum_{v \in s \cup R_{s}} C_{\text {out }}(v)+(N-1) \cdot \sum_{h \in H_{s}} \min \left[C_{\text {in }}(h), \frac{1}{N} C_{\text {out }}(h)\right]}{N} . \\
& \text { We also let } \eta=\frac{\sum_{r \in R_{s}} C_{\text {out }}(r)}{N-1}+\sum_{h \in H_{s}} \min \left[C_{\text {in }}(h), \frac{1}{N} C_{\text {out }}(h)\right] \\
& \text { and } \theta=\min \left[C_{\text {out }}(s), \min _{r \in R_{s}} C_{\text {in }}(r), \delta\right] . \text { Then we have the } \\
& \text { following results. }
\end{aligned}
$$

Lemma 1. Given a single-source multicast scenario $\mathcal{M}=$ $\left(s, R_{s}, H_{s}, G\right.$, full-mesh), multicast rate $\theta$ can be achieved by packing at most $|V|=1+\left|R_{s}\right|+\left|H_{s}\right|$ routing trees with each depth at most 2 . The optimal multicast rate achieved by routing is at least $\theta$.

Proof: We now show the achievability of $\theta$ by giving explicit constructions of routing trees. These routing trees can be divided into three types $[7,11]$ :

1) One depth-1 tree rooted at $s$ and reaching all receivers in $R_{s}$ over a single hop, i.e., the type-1 tree in Fig.3.

2) $\left|R_{s}\right|$ depth-2 trees, each rooted at $s$, reaching another receiver $r \in R_{s}$ over a first hop, and then reaching all other receivers in $R_{s}-\{r\}$ over a second hop, i.e., the type-2 tree in Fig.3.

3) $\left|H_{s}\right|$ depth-2 trees, each rooted at $s$, and reaching all receivers in $R_{s}$ via different $h \in H_{s}$, i.e., the type-3 tree in Fig.3.

For convenience, let $C_{\min }=\min _{r \in R_{s}} C_{i n}(r)$. We have the following constructions of routing trees.

Case 1: $C_{\min } \leq \min \left[C_{\text {out }}(s), \delta\right]$ and $C_{\min } \leq \eta$.

In this case, $\theta=C_{\text {min }}$. We need $\left|R_{s}\right|$ type-2 trees and $\left|H_{s}\right|$ type- 3 trees. First, for each type- 2 tree reaching $r \in R_{s}$ over the first hop, the rate of tree is $\left[C_{\text {out }}(r) \cdot C_{\min }\right] /[(N-1) \cdot \eta]$. Second, for each type-3 tree reaching $h \in H_{s}$ over the first hop, the rate of tree is $\left(\min \left[C_{\text {in }}(h), \frac{1}{N} C_{\text {out }}(h)\right] \cdot C_{\min }\right) / \eta$.

By packing these trees, we see that all nodes in $R_{s}$ have the same rate $C_{m i n}$. Thus $\theta=C_{m i n}$ is achievable by packing $\left|R_{s}\right|+\left|H_{s}\right|$ trees each with depth at most two.

Case 2: $C_{\min } \leq \min \left[C_{\text {out }}(s), \delta\right]$ and $C_{\min }>\eta$.

In this case, $\theta=C_{\min }$. We need one type-1 tree, $\left|R_{s}\right|$ type2 trees and $\left|H_{s}\right|$ type- 3 trees. First, for the only type- 1 tree, the rate of tree is $C_{\min }-\eta$. Second, for each type- 2 tree reaching $r \in R_{s}$ over the first hop, the rate of tree is $C_{\text {out }}(r) /(N-1)$. 
Third, for each type- 3 tree reaching $h \in H_{s}$ over the first hop, the rate of tree is $\min \left[C_{\text {in }}(h), \frac{1}{N} C_{\text {out }}(h)\right]$.

By packing these trees, we see that all nodes in $R_{s}$ have the same rate $C_{\min }$. Thus $\theta=C_{\min }$ is achievable by packing $1+\left|R_{s}\right|+\left|H_{s}\right|$ trees with each depth at most two.

Case 3: $C_{\text {out }}(s) \leq \min \left[C_{\text {min }}, \delta\right]$.

In this case, $\theta=C_{\text {out }}(s)$. Then we have $C_{\text {out }}(s) \leq$ $\min \left[C_{\min }, \eta\right]$. We need $\left|R_{s}\right|$ type- 2 trees and $\left|H_{s}\right|$ type-3 trees. First, for each type-2 tree reaching $r \in R_{s}$ over the first hop, the rate of tree is $\left[C_{\text {out }}(r) \cdot C_{\text {out }}(s)\right] /[(N-1) \cdot \eta]$. Second, for each type-3 tree reaching $h \in H_{s}$ over the first hop, the rate of tree is $\left(\min \left[C_{\text {in }}(h), \frac{1}{N} C_{\text {out }}(h)\right] \cdot C_{\text {out }}(s)\right) / \eta$.

By packing these trees, we see that all nodes in $R_{s}$ have the same rate $C_{\text {out }}(s)$. Thus $\theta=C_{\text {out }}(s)$ is achievable by packing $\left|R_{s}\right|+\left|H_{s}\right|$ trees ,each with depth at most two.

Case 4: $\delta \leq \min \left[C_{\text {out }}(s), C_{\min }\right]$.

In this case, $\theta=\delta$. Then we have $C_{\text {out }}(s)>\eta$. We need one type- 1 tree, $\left|R_{s}\right|$ type- 2 trees and $\left|H_{s}\right|$ type- 3 trees. First, for the only type- 1 tree, the rate of tree is $\left[C_{\text {out }}(s)-\eta\right] / N$. Second, for each type-2 tree reaching $r \in R_{s}$ over the first hop, the rate of tree is $C_{\text {out }}(r) /(N-1)$. Third, for each type3 tree reaching $h \in H_{s}$ over the first hop, the rate of tree is $\min \left[C_{\text {in }}(h), \frac{1}{N} C_{\text {out }}(h)\right]$.

By packing these trees, we see that all nodes in $R_{s}$ have the same rate $\delta$. Thus $\theta=\delta$ is achievable by packing $1+\left|R_{s}\right|+$ $\left|H_{s}\right|$ trees, each with depth at most two.

So the optimal multicast rate achieved by routing is at least $\theta$. This concludes the proof.

Lemma 2. Given a single-source multicast scenario $\mathcal{M}=$ $\left(s, R_{s}, H_{s}, G\right.$, full-mesh). The helper nodes satisfying the condition (3). For any achievable multicast rate $z$, let $f_{z}^{*}(E)$ be an irreducible edge rate vector with respect to $z$. Then the sum of outgoing edge rates of all helpers is no more than $N$ times the sum of incoming edge rates of all helpers, i.e.,

$$
\sum_{h \in H_{s}} \sum_{e \in O u t(h)} f_{z}^{*}(e) \leq N \cdot \sum_{h \in H_{s}} \sum_{e \in \operatorname{In}(h)} f_{z}^{*}(e) .
$$

We also have an upper bound on the the sum of outgoing edge rates of all helpers, i.e.,

$$
\sum_{h \in H_{s}} \sum_{e \in O u t(h)} f_{z}^{*}(e) \leq N \cdot \sum_{h \in H_{s}} \min \left[C_{\text {in }}(h), \frac{1}{N} C_{\text {out }}(h)\right]
$$

Proof: We first prove (4) by contradiction. Suppose (4) is not true, then by the node upload capacity constraint (1),

$$
\sum_{h \in H_{s}} C_{\text {out }}(h) \geq \sum_{h \in H_{s}} \sum_{e \in \text { Out }(h)} f_{z}^{*}(e)>N \cdot \sum_{h \in H_{s}} \sum_{e \in \operatorname{In}(h)} f_{z}^{*}(e)
$$

On the other hand, by the node download capacity constraint (2), we have

$$
\sum_{h \in H_{s}} \sum_{e \in \operatorname{In}(h)} f_{z}^{*}(e) \leq \sum_{h \in H_{s}} C_{i n}(h) .
$$

Thus by (6), (7) and (3),

$$
\begin{aligned}
\sum_{h \in H_{s}} \sum_{e \in I n(h)} f_{z}^{*}(e) & <\min \left[\sum_{h \in H_{s}} C_{\text {in }}(h), \frac{\sum_{h \in H_{s}} C_{\text {out }}(h)}{N}\right] \\
& =\sum_{h \in H_{s}} \min \left[C_{\text {in }}(h), \frac{C_{\text {out }}(h)}{N}\right] .
\end{aligned}
$$

We now show that $f_{z}^{*}(E)$ is reducible by constructing a new edge rate vector $f_{z}^{\prime}(E)$ as follows:

- For all $e$ between nodes in source and receivers, i.e., $R_{s} \cup$ $\{s\}$, let $f_{z}^{\prime}(e)=f_{z}^{*}(e)$

- For all $e$ between nodes in $H_{s}$, let $f_{z}^{\prime}(e)=0$

- The total amount of information from $R_{s} \cup\{s\}$ to $H_{s}$ is kept the same as $\sum_{h \in H} \sum_{e \in \operatorname{In}(h)} f_{z}^{*}(e)$. In particular, for each helper $h \in H_{s}$, the sum of total incoming edge rates is chosen to be at most $\min \left[\frac{1}{N} C_{\text {out }}(h), C_{\text {in }}(h)\right]$. Such an operation is feasible by (9). Each helper node then replicates the contents it receives from node $r$ in $R_{s} \cup\{s\}$, and broadcasts to all other nodes in $R_{s} \cup\{s\}$.

By above steps, all receivers with edge rate vector $f_{z}^{\prime}(E)$ have the same information from helper nodes in $H_{s}$ as with edge rate vector $f_{z}^{*}(E)$. Thus rate $z$ are still achievable by $f_{z}^{\prime}(E)$. One possible scheme involves using a Maximum Distance Separable (MDS) code to generate distinct contents for each helper. Then the MDS property guarantees decodability. Note that helper nodes do not need to code, but the source and receivers do. This scheme's encoding operations might be drastically different from those of the original scheme used in the assignment $f_{z}^{*}(E)$.

We thus have

$$
\begin{aligned}
\sum_{h \in H_{s}} \sum_{e \in \text { Out }(h)} f_{z}^{\prime}(e) & =N \cdot \sum_{h \in H_{s}} \sum_{e \in \operatorname{In}(h)} f_{z}^{*}(e) \\
& <\sum_{h \in H_{s}} \sum_{e \in \text { Out }(h)} f_{z}^{*}(e)
\end{aligned}
$$

On the other hand, for all $r \in R_{s} \cup\{s\}$, the information sent out by $r$ is unchanged, so

$$
\sum_{r \in R_{s} \cup\{s\}} \sum_{e \in O u t(r)} f_{z}^{\prime}(e)=\sum_{r \in R_{s} \cup\{s\}} \sum_{e \in O u t(r)} f_{z}^{*}(e) .
$$

Summing all outgoing edge rates of every node for $f_{z}^{\prime}(E)$ and $f_{z}^{*}(E)$, we have

$$
\sum_{v \in V} \sum_{e \in O u t(v)} f_{z}^{\prime}(e)<\sum_{v \in V} \sum_{e \in O u t(v)} f_{z}^{*}(e) .
$$

Thus, $f_{z}^{*}(E)$ is reducible, leading to a contradiction, and therefore proving (4).

Now, we will prove (5). By (4) and (7), we have

$$
\begin{aligned}
\sum_{h \in H_{s}} \sum_{e \in \text { Out }(h)} f_{z}^{*}(e) & \leq N \cdot \sum_{h \in H_{s}} \sum_{e \in \operatorname{In}(h)} f_{z}^{*}(e) \\
& \leq N \cdot \sum_{h \in H_{s}} C_{i n}(h) .
\end{aligned}
$$


On the other hand, by (1),

$$
\sum_{h \in H_{s}} \sum_{e \in O u t(h)} f_{z}^{*}(e) \leq \sum_{h \in H_{s}} C_{\text {out }}(h) .
$$

Therefore, with (3), we have

$$
\begin{aligned}
\sum_{h \in H_{s}} \sum_{\text {ein Out }(h)} f_{z}^{*}(e) & \leq \min \left[N \cdot \sum_{h \in H_{s}} C_{\text {in }}(h), \sum_{h \in H} C_{\text {out }}(h)\right] \\
& =N \cdot \min \left[\sum_{h \in H_{s}} C_{\text {in }}(h), \frac{\sum_{h \in H_{s}} C_{\text {out }}(h)}{N}\right] \\
& =N \cdot \sum_{h \in H_{s}} \min \left[C_{\text {in }}(h), \frac{1}{N} C_{\text {out }}(h)\right]
\end{aligned}
$$

Now we begin the proof of theorem 4 .

Proof: Let the optimal multicast rate achievable by network coding be denoted by $z^{*}$. The corresponding irreducible edge rate vector is denoted by $f_{z}^{*}(E)=\left[f_{z}^{*}(e), e \in E\right]$. We now prove $z^{*}=\theta$.

First, by the cut-set condition on source and receivers, we obtain the following bounds on the multicast rate $z^{*}$ :

$$
\begin{aligned}
& z^{*} \leq C_{\text {out }}(s) . \\
& z^{*} \leq \min _{r \in R_{s}} C_{\text {in }}(r) .
\end{aligned}
$$

Further, focusing on the cut separating $R_{s}$ from $V-R_{s}$, we have

$$
\begin{aligned}
N & \cdot z^{*} \leq \sum_{r \in R_{s}} \sum_{e \in \operatorname{In}(r)} f_{z}^{*}(e) \\
& =\sum_{v \in V} \sum_{e \in \operatorname{In}(v)} f_{z}^{*}(e)-\sum_{h \in H_{s}} \sum_{e \in \operatorname{In}(h)} f_{z}^{*}(e) \\
& =\sum_{v \in V} \sum_{e \in O u t(v)} f_{z}^{*}(e)-\sum_{h \in H_{s}} \sum_{e \in \operatorname{In}(h)} f_{z}^{*}(e) \\
& \leq \sum_{v \in s \cup R_{s}} C_{\text {out }}(v)+\sum_{h \in H_{s}} \sum_{e \in O u t(h)} f_{z}^{*}(e)-\sum_{h \in H_{s}} \sum_{e \in \operatorname{In}(h)} f_{z}^{*}(e)
\end{aligned}
$$

By Lemma 2 we have

$$
\begin{aligned}
& N \cdot z^{*} \leq \sum_{v \in s \cup R_{s}} C_{\text {out }}(v)+(1-1 / N) \cdot \sum_{h \in H_{s}} \sum_{e \in \text { Out }(h)} f_{z}(e) \\
& \leq \sum_{v \in s \cup R_{s}} C_{\text {out }}(v)+(N-1) \cdot \sum_{h \in H_{s}} \min \left[C_{\text {in }}(h), \frac{1}{N} C_{\text {out }}(h)\right]
\end{aligned}
$$

Thus with (10), (11) and (13), we have $z^{*} \leq \theta$. Since routing is a special case of network coding, $\theta \leq z^{*}$. Thus $z^{*}=\theta$. By Lemma 1, the optimal multicast rate is achievable by packing at most $|V|=1+\left|R_{s}\right|+\left|H_{s}\right|$ trees with each depth at most two. Therefore, routing is rate-optimal.

\section{CONCLUSIONS}

In this paper, we view node-capacitated network through two lenses. First, we focus on the difference between coding and routing, and on teasing out scenarios where routing suffices to attain the optimal rate region. For many interesting scenarios, this turn out to be the case. Second, we focus on seeing whether cut-set bounds on rate region are tight by considering special classes of networks.

\section{ACKNOWLEDGMENT}

The authors would like to thank Minghua Chen for helpful discussions. Work of the second author was partially supported by GRF 412608. Work of the third author was supported in part by CERG grants CUHK4231/04E, 414005, 413806, 414307, and Collaborative Research Fund CUHK2/06C from the RGC of the HKSAR, China, as well as the NSFC-RGC joint research grant NCUHK411/07.

\section{REFERENCES}

[1] R. Alswede, N. Cai, S.-Y. R. Li, and R. W. Yeung, "Network information flow," IEEE Trans. Information Theory, vol.46, no.4, pp. 1204-1216, July 2000.

[2] S. Li, R.Yeung and N. Cai, "Linear network Coding," IEEE Trans. Information Theory, vol.49, no.2, pp.371381, Feb 2003.

[3] S. Jaggi, P. Sanders, P. A. Chou, M. Effros, S. Egner, K. Jain, and L. Tolhiuzen, "Polynomial time algorithms for multicast network code construction," IEEE Trans. Inform. Theory, vol. 51, no.6, pp. 1973-1982, 2005.

[4] L.R. Ford, Jr., and D.R.Fulkerson, Flows in Networks. Princeton University Press, 1962.

[5] J. Edmonds, "Edge-disjoint branchings," Combinatorial Algorithms, R. Rustin, ed., pp.91-96, 1973.

[6] J. Mundinger, R. Weber, and G. Weiss, "Analysis of peer-to-peer file dissemination amongst users of different upload capacities," IFIP Performance 2005.

[7] J. Li, P.A. Chou, C. Zhang, "Mutualcast: an efficient mechanism for one-to-many content distribution," $A C M$ Sigcomm Asia Workshop, Beijing, China, April 2005.

[8] D.M. Chiu, R.W. Yeung, J.Q. Huang and B. Fan, "Can network coding help in P2P networks," NetCod 2006.

[9] R. Kumar, Y. Liu, K.W. Ross, "Stochastic fluid theory of P2P streaming systems," IEEE INFOCOM 2007.

[10] L. Massoulié, A. Twigg, C. Gkantsidis, and P. Rodriguez, "Randomized decentralized broadcasting algorithms," IEEE INFOCOM 2007.

[11] M. Chen, M. Ponec, S. Sengupta, J. Li and P.A. Chou, "Utility maximization in peer-to-peer systems," ACM Sigmetrics 2008.

[12] S. Sengupta, M. Chen, P.A. Chou and J. Li, "On optimality of routing for multi-source multicast communication scenarios with node uplink constraints," ISIT 2008.

[13] (http://personal.ie.cuhk.edu.hk/ zyshao6/). Z. Shao, S. Jaggi and S.Y. Robert Li, To code, or not to code: rate optimality in node-Capacitated networks, CUHK Technical Report, Jan 2009. 